\title{
BMJ Open Which orthopaedic trauma patients are likely to refuse to participate in a clinical trial? A latent class analysis
}

\author{
Nathan N O'Hara (D) , ${ }^{1}$ Yasmin Degani, ${ }^{1}$ Debra Marvel, ${ }^{2}$ David Wells, ${ }^{2}$ \\ C Daniel Mullins, ${ }^{3}$ Stephen Wegener, ${ }^{4}$ Katherine Frey, ${ }^{5}$ Tara Joseph, ${ }^{5}$ \\ Jonathan Hurst, ${ }^{1}$ Renan Castillo, ${ }^{5}$ Robert V O'Toole, ${ }^{1}$ on behalf of PREVENT CLOT \\ Stakeholder Committee
}

To cite: O'Hara NN, Degani Y, Marvel D, et al. Which orthopaedic trauma patients are likely to refuse to participate in a clinical trial? A latent class analysis. BMJ Open 2019;9:e032631. doi:10.1136/ bmjopen-2019-032631

- Prepublication history for this paper is available online. To view these files, please visit the journal online (http://dx.doi org/10.1136/bmjopen-2019032631).

Received 28 June 2019 Revised 27 August 2019 Accepted 23 September 2019

Check for updates

(C) Author(s) (or their employer(s)) 2019. Re-use permitted under CC BY-NC. No commercial re-use. See rights and permissions. Published by BMJ.

For numbered affiliations see end of article.

Correspondence to Mr Nathan N O'Hara; nohara@som.umaryland.edu

\section{ABSTRACT}

Objective The study aimed to assess systematic differences in the characteristics of patients that consented for the trial compared with the broader pool of eligible patients in a large, pragmatic orthopaedic trauma trial.

Design A retrospective observational study performed from April 2017 to March 2018.

Setting Academic trauma centre in Baltimore, USA. Participants There were 642 eligible adult trial participants with an operative fracture to the appendicular skeleton and were indicated for blood clot prophylaxis. The median age of the sample was 50 years (IQR: 31-63), and $60 \%$ were male.

Primary outcome measure The primary outcome was the refusal to enrol in the trial. Demographic and injury covariates were included in iterations of latent class models. The final model was selected based on a minimum Bayesian information criterion.

Results The final model identified three clusters with five covariates predictive of cluster membership (age, neighbourhood-based socioeconomic status, alcohol use, multiple fractures, multiple surgeries). The three clusters were associated with 22\% (Cluster 1), 38\% (Cluster 2) and $62 \%$ (Cluster 3) refusal rates, respectively. Members of Cluster $3(n=84)$ were most commonly between 66 and 80 years of age ( $49 \%$ vs $6 \%$ (Cluster 1 ) and $21 \%$ (Cluster 2)), of high neighbourhood-based socioeconomic status ( $85 \%$ vs $63 \%$ (Cluster 1 ) and $8 \%$ (Cluster 2)), with isolated fractures (100\% vs $80 \%$ (Cluster 1) and $92 \%$ (Cluster 2)), and were less likely to have multiple surgeries compared with the other clusters (28\% vs $47 \%$ (Cluster 1 ) and $35 \%$ (Cluster 2)).

Conclusion In this study, the likelihood of refusing to participate in the trial ranged from $22 \%$ to $62 \%$ in the three identified clusters. Elderly age, high socioeconomic status, and less severe injuries defined the cluster that was most likely to refuse trial participation.

Trial registration number NCT02984384.

\section{INTRODUCTION}

In clinical research, randomised controlled trials (RCTs) are considered the gold standard in evaluating the effectiveness of treatments. However, lags in enrolling participants
Strengths and limitations of this study

- A nine-member patient stakeholder advisory committee was instrumental in the design of this study and the interpretation of the study findings.

- The study uses latent class analysis to investigate the systematic differences in patients based on their participation in a clinical trial.

- This study adds to a paucity of data on factors associated with clinical trial participation, particularly in the trauma population.

- The study was only performed at one centre and may have limited generalisability to other centres and other trials.

- Over half of participants that refused to participate did not provide a reason for this refusal, limiting our ability to compare reasons for refusals with cluster membership.

can delay the availability of evidence, increase the costs of the research, and compromise the generalisability of results. ${ }^{1}$ Identified barriers to enrolment can include challenges at the site level, including fewer eligible participants, competing studies, or lack of a clinical equipoise, but can also include patient levels factors, such as the perceived burden of the research, stress due to randomisation, a propagation of questionnaires and medical testing, and additional clinical visits. ${ }^{23}$ The high refusal rate observed in many clinical trials, particularly given the systematic differences between study participants and the eligible study population, has the potential to impact the external validity of the research findings.

The reported refusal rates into randomised orthopaedic trials typically range from $8 \%$ to $20 \%$, although this rate can decline to more than $50 \%$ when surgical interventions are compared with non-surgical interventions. ${ }^{4}$ One such study reported a refusal rate of more than $90 \% .^{5}$ These rates of trial refusal 
are consistent with the oncology trial literature, for which an estimated $45 \%-95 \%$ of eligible adult patients declined participation in the clinical trial, with higher refusal rates for racial and ethnic minorities. ${ }^{6-8}$ A review of nearly 300 cardiology trials found median refusal rates to be less than $17 \%$. Participation rates were the highest when consent occurred in the acute care setting, for studies conducted outside the USA, and in studies sponsored by industry. ${ }^{9}$ In surgical RCTs, refusal rates have been found to be higher among participants who are older, of a minority group, and more ill, while evidence regarding the impact of education varies. ${ }^{10}$ While the disparities in research participation have been investigated in multiple clinical areas of research, there is a paucity of data specific to patients with traumatic injuries. Despite the psychological distress associated with a traumatic injury, ${ }^{11}$ refusal rates for trauma trials may be much lower than what has been reported in oncology literature. Several recently completed trials of orthopaedic trauma patients reported refusal rates of less than $15 \% .^{12-14}$

A large, pragmatic trial comparing aspirin versus low molecular weight heparin to prevent blood clots in orthopaedic trauma patients presented an opportunity to assess systematic differences in the characteristics of patients that consented for the trial compared with the broader pool of eligible patients. The study aimed to assess systematic differences in the characteristics of patients that consented for the trial compared with the broader pool of eligible patients in a large, pragmatic orthopaedic trauma trial. The secondary objective of this study was to resolve if the patient's reason for refusal was predictable based on these contributing factors. Based on the surgery and oncology literature, ${ }^{210}$ we hypothesised that patients of ethnic minorities, of lower socioeconomic status, and elderly patients would be more likely to refuse participation in the clinical trial.

\section{METHODS}

\section{Study design}

In this retrospective cohort study, we used a latent class analysis to identify patient factors predictive of trial participation. Latent class analysis can be used to identify unobserved groups, or clusters, in a dataset, which can be described based on observed parameters. ${ }^{15}$ The PREVENTion of CLots in Orthopaedic Trauma (PREVENT CLOT): A Randomised Pragmatic Trial Comparing the Complications and Safety of Blood Clot Prevention Medicines Used in Orthopaedic Trauma Patients is a trial currently being conducted at 21 sites in the USA and Canada. This study linked 1 year of PREVENT CLOT screening data (April 2017-March 2018) from a single clinical site, a large academic trauma centre, with hospital records to extract demographic and injury characteristics on all eligible participants.

\section{Study participants}

Patients were eligible for inclusion in PREVENT CLOT if they: (1) were 18 years of age or older, (2) had a planned operative or non-operative pelvis or acetabular fracture, or any operative extremity fracture proximal to the metatarsals or metacarpals, and (3) were at an increased risk of a blood clot from their orthopaedic injury and would receive a prophylactic blood thinner regimen per standard of care. Patients were excluded if they: (1) presented to the hospital more than 48 hours from injury, (2) had received more than two doses of venous thromboembolism (VTE) prophylaxis, (3) were on long term blood thinners, (4) had a VTE within the last 6 months, (5) had an allergy to the study medications, (6) had an underlying chronic clotting disorder, (7) had end-stage renal disease, (8) were pregnant or lactating, (9) did not speak either English or Spanish, or (10) were a prisoner at the time of screening.

By virtue of the types of injuries studied, often resulting from high energy mechanisms such as motor vehicle crashes, high falls, and blast injuries, some eligible patients were unable to provide consent for the study due to associated traumatic brain injury or intubation status. Including these patients was integral to ensuring the generalisability of the research findings. However, in these situations, the research staff member sought consent from a legally authorised representative.

If the patient met the eligibility criteria, a member of the research staff would approach the eligible participants or their legally authorised representatives to discuss the study and request consent to participate. The research staff endeavoured to answer all questions posed by the eligible study participants and their caregivers to ensure an understanding of the protocol. After introducing the study and reviewing the consent form, the research staff member posed several questions to assess the participant's (or representative's) comprehension of the study, the responsibilities of participation, and alternatives to participation.

\section{Primary outcome and included covariates}

The primary outcome was the refusal to enrol in the PREVENT CLOT trial. Several covariates were considered as predictors of cluster membership in iterations of the latent class analysis. Participant age was categorised as 40 years and under, $41-65$ years, 66-80 years, and over 80 years of age. The race of study participants was distinguished as white, African-American, Asian, or a fourth 'other' category. We also included diabetic status, frequent alcohol use, hypertension, and smoking status (defined as a current smoker, former smoker, or never smoker). Health insurance was coded as employer-based insurance, Medicaid, Medicare and Tricare. The injuries were classified by upper versus lower extremity fractures, which included pelvis and acetabulum fractures. The number of fractures and the number of surgeries were also reported. Finally, socioeconomic status was determined by converting the patient's ZIP +4 code of their home address in the hospital records to an Area Deprivation Index (ADI) value. ${ }^{16}$ ADI was developed by the Health 
Resources and Services Administration and represents a geographic area-based measure of the socioeconomic status experienced by a neighbourhood as a composite score of 17 variables, including income disparity and percent of the population aged 25 and older with at least a high school diploma. For this study, ADI values were stratified into quintiles. Missing values for ADI $(n=6)$ were imputed using multiple imputation. ${ }^{17}$ While not included in developing the latent classes, the patient's reason for refusing to participate in the trial was recorded and used in the secondary analysis.

\section{Statistical analysis}

In the latent class analysis, a sequence of models, from one to 10 clusters, were indexed with various iterations of candidate covariates to determine the optimal model based on a minimum Bayesian Information Criterion (BIC).$^{18}$ The conditional probability of cluster membership for each included covariate was reported. We used $\chi^{2}$ tests to determine the association between cluster membership and the documented reason for the refusal to participate in the trial.

\section{Sample size}

While there is no formally agreed on sample size calculation for latent class analyses, several parameters influence the accuracy of the estimates. These parameters include the prevalence of the outcome, the number of items included in the analysis, the number of clusters to be fit, and the interclass distances between the clusters. ${ }^{19}$ Based on simulation modelling by Tein $e t$ al and the prevalence of our outcome $(36 \%),{ }^{19}$ the study's samples size of over 600 eligible study participants was estimated to provide $80 \%$ power for a model including five or fewer clusters, 15 or fewer predictor covariates, an interclass distances of Cohen's $d \leq 0.8$, and model selection based on a minimum BIC.

\section{Patient and public involvement}

The patient stakeholder advisory committee of the PREVENT CLOT trial includes trauma patients, caregivers, members of patient advocacy organisations, clinicians, as well as payor representatives. The members of the advisory committee were involved in the interpretation of the findings, critical revision of the manuscript, and translating the conclusions into improved recruitment practices for the trial.

\section{RESULTS}

Of the 642 patients that were eligible for participation in the trial, 233 patients $(36 \%)$ refused participation. The median age of the sample was 50 years (IQR: $31-63$ ), and $60 \%$ were male (table 1 ). Two-thirds of the sample was white, and $30 \%$ were African-American. Over half of the sample reported regular alcohol use $(\mathrm{n}=335), 30 \%$ were hypertensive, $28 \%$ were current smokers, and $12 \%$ were diabetic. Seventy-one per cent of the eligible participants
Table 1 Description of patient characteristics $(n=642)$

Refused

\begin{tabular}{lrr} 
Characteristic & N (\%) & (\%) \\
\hline $\begin{array}{l}\text { Age, years } \\
<40\end{array}$ & $252(39)$ & 33 \\
$41-65$ & $254(40)$ & 30 \\
$66-80$ & $97(15)$ & 51 \\
$>80$ & $39(6)$ & 59 \\
Sex & & \\
Male & $382(60)$ & 32 \\
Female & $260(40)$ & 42 \\
Race & & \\
White & $430(67)$ & 36 \\
African-American & $186(29)$ & 33 \\
Asian & $9(1)$ & 55 \\
Other & $17(3)$ & 53
\end{tabular}

Smoking status

\begin{tabular}{|c|c|c|}
\hline Current & $180(28)$ & 28 \\
\hline Former & $142(22)$ & 38 \\
\hline Never & $320(50)$ & 40 \\
\hline Hypertension & $193(30)$ & 41 \\
\hline Alcohol use & $335(52)$ & 31 \\
\hline Diabetes & $75(12)$ & 36 \\
\hline \multicolumn{3}{|c|}{$\begin{array}{l}\text { Neighbourhood-based } \\
\text { socioeconomic status (quintiles) }\end{array}$} \\
\hline 1 (highest) & $204(32)$ & 42 \\
\hline 2 & $205(32)$ & 38 \\
\hline 3 & $95(15)$ & 35 \\
\hline 4 & $68(11)$ & 24 \\
\hline 5 (lowest) & $67(11)$ & 30 \\
\hline \multicolumn{3}{|l|}{ Type of health insurance } \\
\hline Employer-based & $452(71)$ & 35 \\
\hline Medicare & $122(19)$ & 46 \\
\hline Medicaid & $43(7)$ & 19 \\
\hline Uninsured & $21(3)$ & 40 \\
\hline Tricare & $4(1)$ & 50 \\
\hline \multicolumn{3}{|l|}{ Fracture location } \\
\hline Upper extremity & $398(62)$ & 37 \\
\hline $\begin{array}{l}\text { Lower extremity or pelvis/ } \\
\text { acetabulum }\end{array}$ & $244(38)$ & 35 \\
\hline Multiple fractures & $81(13)$ & 22 \\
\hline Multiple surgeries & $254(40)$ & 30 \\
\hline
\end{tabular}

had employer-based insurance, and one-third lived in a top socioeconomic quartile neighbourhood, based on the ADI.

Based on a minimum BIC, the final model identified three clusters with five covariates predictive of cluster membership (table 2). The predictors included age, 
Table 2 Probability of membership to one of three identified clusters

\begin{tabular}{lccll}
\hline & Cluster 1 & Cluster 2 & Cluster 3 & $\begin{array}{l}\text { Total } \\
\text { (\%) }\end{array}$ \\
\hline $\begin{array}{l}\text { Probability } \\
\text { of refusing } \\
\text { participation }\end{array}$ & $22 \%$ & $38 \%$ & $62 \%$ & 36 \\
\hline$N$ & & & & \\
\hline Characteristic & & 382 & 84 & \\
\hline Age & & & & \\
\hline$>80$ & 176 & $16 \%$ & $12 \%$ & 6 \\
\hline $66-80$ & $5 \%$ & $5 \%$ & $37 \%$ & $\mathbf{1 5}$ \\
\hline $41-65$ & $46 \%$ & $42 \%$ & $28 \%$ & 40 \\
\hline$<40$ & $48 \%$ & $36 \%$ & $24 \%$ & 39 \\
\hline Socioeconomic status (quintile) & & & \\
\hline 1 (highest) & $32 \%$ & $2 \%$ & $42 \%$ & $\mathbf{3 2}$ \\
\hline 2 & $31 \%$ & $6 \%$ & $43 \%$ & $\mathbf{3 2}$ \\
\hline 3 & $13 \%$ & $49 \%$ & $6 \%$ & $\mathbf{1 5}$ \\
\hline 4 & $11 \%$ & $25 \%$ & $5 \%$ & $\mathbf{1 1}$ \\
\hline 5 (lowest) & $14 \%$ & $17 \%$ & $4 \%$ & $\mathbf{1 1}$ \\
\hline Alcohol use & $71 \%$ & $3 \%$ & $36 \%$ & $\mathbf{5 2}$ \\
\hline Multifracture & $20 \%$ & $8 \%$ & $0 \%$ & $\mathbf{1 3}$ \\
\hline Multiple surgeries & $47 \%$ & $35 \%$ & $28 \%$ & $\mathbf{4 0}$ \\
\hline
\end{tabular}

neighbourhood-based socioeconomic status, alcohol use, multifracture injuries, and having multiple surgeries. The three clusters were associated with 22\% (Cluster 1), 38\% (Cluster 2\%) and 62\% (Cluster 3) refusal rates, respectively. Members of Cluster $3(n=84)$, who were most likely to refuse participation in the study, were most commonly between 66 and 80 years of age, of high socioeconomic status (first or second quintile), with isolated fractures, and were less likely to have multiple surgeries compared with the other clusters. Members of Cluster 2 (n=382) had the highest probability of being in the lower third or fourth neighbourhood-based socioeconomic quintile and had a low likelihood of alcohol use. Members of Cluster $1(n=176)$ had a high probability of alcohol use, multiple fractures and multiple surgeries.

When refusing to participate in the trial, over half $(55 \%)$ of the eligible participants did not provide a reason for their refusal (table 3). Thirty-five per cent of eligible participants that refused participation expressed a lack of interest in research, while $21 \%$ stated a treatment preference. Members of Cluster 2 were most likely to refuse participation due to a fear of medication side effects $(p<0.01)$ or have their legally authorised representative refuse participation $(\mathrm{p}=0.04)$. Refusal due to a preference for the treating surgeon to select their treatment was only reported by members of Clusters 2 and $3(\mathrm{p}=0.16)$.

\section{DISCUSSION}

In this study, we identified three clusters of eligible trial participants, and their likelihood of refusing to participate in the trial, based on observed characteristics. The cluster of eligible study participants most likely to refuse participation in the trial had members that were commonly between 66 and 80 years of age, of higher neighbourhood-based socioeconomic status, and sustained less severe injuries. The cluster of eligible study participants most likely to consent for the trial were more likely to have multiple fractures and multiple surgeries. In the majority of the refusals, eligible participants would not provide a reason for their refusal or expressed a general lack of interest in research. However, patient preferences for a treatment arm and lack of interest in participation was consistent with reasons for refusal reported in the surgical literature. ${ }^{10}$

The overall rate of refusal of $36 \%$ was much lower than what has been reported in the oncology literature, ${ }^{6-8}$ but higher than the refusal rates observed in previous surgery or orthopaedic research, ${ }^{49}$ and nearly double the rates of recent large orthopaedic trauma trials. ${ }^{12-14}$ The previous surgery, orthopaedic, and orthopaedic trauma trials compared interventions specific to the surgical

\begin{tabular}{|c|c|c|c|c|c|}
\hline Refusal reason & Overall (\%) & Cluster $1(\%)$ & Cluster $2(\%)$ & Cluster $3(\%)$ & $P$ value \\
\hline Lack of interest & 35 & 35 & 35 & 35 & 0.99 \\
\hline Treatment preference & 21 & 26 & 12 & 21 & 0.52 \\
\hline Caregiver resistance & 13 & 19 & 12 & 10 & 0.48 \\
\hline Preferred surgeon to select & 8 & 0 & 12 & 12 & 0.13 \\
\hline Fear of research & 8 & 10 & 6 & 9 & 0.90 \\
\hline Overwhelmed & 8 & 10 & 6 & 9 & 0.90 \\
\hline Fear of side effects & 3 & 0 & 18 & 0 & $<0.01$ \\
\hline LAR refused & 3 & 3 & 12 & 0 & 0.04 \\
\hline In other studies & 2 & 0 & 0 & 3 & 0.43 \\
\hline Follow-up burden & 1 & 0 & 0 & 2 & 0.66 \\
\hline
\end{tabular}

LAR, legally authorised representative. 
procedure. ${ }^{4}{ }^{12-14}$ By contrast, the PREVENT CLOT trial compares prophylactic regimens for prevention of a postoperative complication. Several factors may have led to the higher refusal rates in PREVENT CLOT compared with the other large orthopaedic trauma trials. The PREVENT CLOT trial compares low molecular weight heparin to aspirin for the prevention of blood clots. In the first few days after a fracture, trauma patients likely place great importance on their surgical treatment and direct less attention towards preventing a postoperative complication. Patients may also believe they have more control over preventing a postoperative complication than the nuances of their surgical treatment, and therefore, less likely to leave the prophylactic regimen to chance. Trauma patients, particularly older patients, also likely have some familiarity with aspirin and may have a preference for or against that medication which influences their trial participation decision.

Reviews of clinical trials have found that racial minorities were reported to be less likely to participate in a clinical trial. ${ }^{620}$ By contrast, race was not selected as a predictive covariate in our latent class analysis. The study location was a public hospital located in a neighbourhood largely populated by people of a racial minority. The hospital and its affiliated university have made a concerted effort to integrate with the local community. The similarities in trial participation rates across patients of different races in this study may be a result of these community integration initiatives. Eligible participants that were between 66 and 80 years of age, of higher neighbourhood-based socioeconomic status, and less severely injured, were the most likely to refuse participation. It is possible that wealthy patients may feel more empowered to refuse study participation. The correlation between increased patient age and the probability of refusing to participate in a clinical trial has been previously reported. ${ }^{810}$ In PREVENT CLOT, the informed consent process is administered by research staff who, at this study site, were typically between 20 and 35 years of age. The age gap between the enrolling research staff member and the eligible participant may negatively affect participation. In addition, less severely injured patients may also be less likely to participate in research if they perceive it will delay their discharge.

Members of Cluster 2 were unique in their refusals due to a fear of medication side effects. The higher proportion of patients with a lower neighbourhood-based socioeconomic status in this cluster may align with lower health literacy levels, and is consistent with previous research. ${ }^{20}$ Side effects for low molecular weight heparin include bleeding and irritation from the injections. The side effects due to aspirin are arguably less frequent and more benign. As the VTE prophylaxis standard of care for orthopaedic trauma patients at this site was low molecular weight heparin, participating in the trial gives the participant a chance of receiving a prophylactic medication with a more limited side effect profile.

Members of Cluster 1 were the most likely to have multiple fractures and have multiple surgeries, suggesting they were the most severely injured. Eligible participants were excluded from PREVENT CLOT once they have received the third dose of a VTE prophylaxis. This restriction creates a time pressure to consent. For more severely injured patients, the time pressure often requires a patient's caregiver to be involved in the decision to enrol. Many caregivers may be uncomfortable making the decision to enrol on behalf of the eligible participant and refuse to enrol in the trial on those grounds. Similarly, Cluster 3, and its more elderly members may also have a caregiver involved in study enrolment decision and, similar to Cluster 1, observed refusals by the caregiver.

With an awareness of these identified clusters, there are several modifications to the enrolment procedures that may reduce refusals in the future. Generally, patients are more likely to participate in research when they understand the study, believe that it is important, and feel connected to the information. ${ }^{21}$ For more elderly eligible participants, research staff may want to involve the treating surgeon in the consent process to reduce the generational gap. If a study participant expresses concern regarding medication side effects, it is essential to ensure that the participant is fully aware of both the benefits and harms associated with each medication. In circumstances where the caregiver must make the decision to participate, the consent process should be delayed as long as possible to enable to study participant to be an active participant in this decision.

For many clinical researchers, the decision of an eligible patient to participate in a trial that compares two approved, commonly used treatments and includes detailed follow-up assessments on the patient's post-treatment well-being represents a rational choice. ${ }^{22}$ Consultation with the PREVENT CLOT patient stakeholders in the interpretation of these data highlights an alternative viewpoint. In the days following a traumatic injury, admitted patients must cope with pain, fatigue, anger, hunger and distress. This is a time of immense personal vulnerability. Refusing to participate in a trial may be influenced by that vulnerability, as well as the patient's perceived empowerment, experience and perspective. Members of Cluster 3 had relatively less severe injuries and therefore, may experience less vulnerability-consequently feeling more empowered to refuse trial participation. Cluster 3 members were also more likely to be of higher neighbourhood-based socioeconomic status and presumably less economically vulnerable. Participating in a study may be perceived as a pathway to expensive care a patient may otherwise not be able to afford. In addition, older patients, such as many members of Cluster 3, likely have more experience with the healthcare system than younger patients. More experienced patients are more aware of how arduous medical recovery can be and have greater familiarity with medication side effects. This accumulation of challenging medical experiences can cause an informed shift in a patient's reference for recovery, and thus discourage any encumbrance to recovery, such as participating in a trial. This is an area for future 
exploration in the trauma population through the development of studies of consent processes nested in enrolment into the main study.

These insights into trial participation must be interpreted within the limitations of the study design. While the PREVENT CLOT trial enrols participants at 21 sites, due to limitations in data access and the resources required to extract data on patients that refused participation in the trial, this study only included data from a single centre. Furthermore, there may be characteristics of the treatments and design of PREVENT CLOT that limits the generalisability of the study findings to orthopaedic trauma trials. In this study, socioeconomic status was calculated using the neighbourhood-based ADI. The ADI is a well-established US census-based measure that provides a composite view of socioeconomic disadvantage. ${ }^{23-25}$ However, the socioeconomic status of the individual patient may not be consistent with the socioeconomic disadvantage of their neighbourhood. When refusing to participate in the trial, over half of the eligible participants failed to provide a reason for this refusal, a finding that is consistent with the general surgical literature. ${ }^{10}$ This lack of information on the reason for refusal limited our statistical power to detect differences in the reasons for trial refusal based on cluster membership.

Overall, the refusal rate for the PREVENT CLOT trial at this site was $36 \%$. Of the three identified clusters, refusal rates ranged from $22 \%$ to $62 \%$. Only five of the 12 candidate covariates were found to be predictive of cluster membership based on the best model fit. These findings suggest that there are limited systematic differences between patients that enrol versus decline to participate in the PREVENT CLOT trial, at this site. While these findings are promising for the generalisability of PREVENT CLOT, they also provide valuable insight towards enhancing the consent experience for eligible patients. Furthermore, the results will inform the design of the recruitment process in future studies.

\section{Author affiliations}

${ }^{1}$ Department of Orthopaedics, University of Maryland School of Medicine, Baltimore, Maryland, USA

${ }^{2}$ PREVENT CLOT Stakeholder Committee, Baltimore, Maryland, USA

${ }^{3}$ Department of Pharmaceutical Health Services Research, University of Maryland

School of Pharmacy, Baltimore, Maryland, USA

${ }^{4}$ Department of Physical Medicine and Rehabilitation, Johns Hopkins University

School of Medicine, Baltimore, Maryland, USA

${ }^{5}$ Department of Health Policy and Management, Johns Hopkins University Bloomberg School of Public Health, Baltimore, Maryland, USA

Acknowledgements The authors thank all members of the orthopaedic trauma research team at the R Adams Cowley Shock Trauma Center for their contributions to this trial.

Collaborators PREVENT CLOT Stakeholder Committee Members (Study Group Name): Stephen Breazeale; Randolph Fenninger; Steven Herndon; Eileen Flores; Stephen Fisher; Michelle Medeiros; Brianna Fowler.

Contributors NNO contributed to the literature search, study design, data analysis, data interpretation, writing and critical revision. YD and $\mathrm{JH}$ contributed to the data collection, data interpretation and critical revision. DM, DW, CDM, SW, TJ and RC contributed to the data interpretation and critical revision. KF contributed to the literature search, data interpretation and critical revision. RVO contributed to the study design, data interpretation and critical revision. All authors have approved the final version of the article submitted and agree to be accountable for all aspects of the work.

Funding Research reported in this manuscript was funded through a PatientCentered Outcomes Research Institute (PCORI) Award (PCS-1511-32745). The views in this publication are solely the responsibility of the authors and do not necessarily represent the views of the PCORI, its board of governors, or methodology committee.

Competing interests None declared.

Patient consent for publication Not required.

Ethics approval The study was reviewed and approved by the University of Maryland School of Medicine Institutional Review Board (HP-00073404).

Provenance and peer review Not commissioned; externally peer reviewed. Data availability statement Data are available upon reasonable request.

Open access This is an open access article distributed in accordance with the Creative Commons Attribution Non Commercial (CC BY-NC 4.0) license, which permits others to distribute, remix, adapt, build upon this work non-commercially, and license their derivative works on different terms, provided the original work is properly cited, appropriate credit is given, any changes made indicated, and the use is non-commercial. See: http://creativecommons.org/licenses/by-nc/4.0/.

ORCID iD

Nathan N 0'Hara http://orcid.org/0000-0003-0537-3474

\section{REFERENCES}

1 Watson JM, Torgerson DJ. Increasing recruitment to randomised trials: a review of randomised controlled trials. BMC Med Res Methodol 2006;6:34.

2 Donovan JL, Paramasivan S, de Salis I, et al. Clear obstacles and hidden challenges: understanding recruiter perspectives in six pragmatic randomised controlled trials. Trials 2014;15:5.

3 Creel AH, Losina E, Mandl LA, et al. An assessment of willingness to participate in a randomized trial of arthroscopic knee surgery in patients with osteoarthritis. Contemp Clin Trials 2005;26:169-78.

4 Lim CT, Roberts HJ, Collins JE, et al. Factors influencing the enrollment in randomized controlled trials in orthopedics. Contemp Clin Trials Commun 2017;8:203-8.

5 Sibai T, Carlisle H. Tornetta P 3rd. The darker side of randomized trials: recruitment challenges. J Bone Joint Surg Am 2012;94:49-55.

6 Brown RF, Cadet DL, Houlihan RH, et al. Perceptions of participation in a phase I, II, or III clinical trial among African American patients with cancer: what do Refusers say? J Oncol Pract 2013;9:287-93.

7 Logan JK, Tang C, Liao Z, et al. Analysis of factors affecting successful clinical trial enrollment in the context of three prospective, randomized, controlled trials. Int J Radiat Oncol Biol Phys 2017:97:770-7.

8 Langford AT, Resnicow K, Dimond EP, et al. Racial/Ethnic differences in clinical trial enrollment, refusal rates, ineligibility, and reasons for decline among patients at sites in the National cancer Institute's community cancer centers program. Cancer 2014;120:877-84.

9 Kerkhoff LA, Butler J, Kelkar AA, et al. Trends in consent for clinical trials in cardiovascular disease. J Am Heart Assoc 2016;5:pii: e003582.

10 Abraham NS, Young JM, Solomon MJ. A systematic review of reasons for nonentry of eligible patients into surgical randomized controlled trials. Surgery 2006;139:469-83.

11 Muscatelli S, Spurr H, O'Hara NN, et al. Prevalence of depression and posttraumatic stress disorder after acute orthopaedic trauma: a systematic review and meta-analysis. J Orthop Trauma 2017;31:47-55.

12 Bhandari M, Jeray KJ, Petrisor BA, et al. A trial of wound irrigation in the initial management of open fracture wounds. $N$ Engl J Med 2015;373:2629-41.

13. Nauth A, Creek AT, Zellar A, et al. Fracture fixation in the operative management of hip fractures (faith): an international, multicentre, randomised controlled trial. Lancet 2017;389:1519-27.

14 Bhandari M, Guyatt G, Walter SD, et al. Randomized trial of reamed and unreamed intramedullary nailing of tibial shaft fractures. J Bone Joint Surg Am 2008;90:2567-78.

15 Lanza ST, Collins LM, Lemmon DR, et al. Proc LCA: a SAS procedure for latent class analysis. Struct Equ Modeling 2007;14:671-94.

16 Kind AJH, Buckingham WR. Making Neighborhood-Disadvantage Metrics Accessible - The Neighborhood Atlas. N Engl J Med 2018;378:2456-8. 
17 Little RJ, Rubin DB. Statistical inference with missing data. 2nd edn. New York, NY: Wiley, 2002.

18 Nylund KL, Asparouhov T, Muthén BO. Deciding on the number of classes in latent class analysis and growth mixture modeling: a Monte Carlo simulation study. Struct Equ Modeling 2007;14:535-69.

19 Tein J-Y, Coxe S, Cham H. Statistical power to detect the correct number of classes in latent profile analysis. Struct Equ Modeling 2013;20:640-57.

20 George S, Duran N, Norris K. A systematic review of barriers and facilitators to minority research participation among African Americans, Latinos, Asian Americans, and Pacific Islanders. Am J Public Health 2014;104:e16-31.

21 Horwood J, Johnson E, Gooberman-Hill R. Understanding involvement in surgical orthopaedic randomized controlled trials: a qualitative study of patient and health professional views and experiences. Int J Orthop Trauma Nurs 2016;20:3-12.

22 Blume LE, Easley D. Learning to be rational. J Econ Theory 1982:26:340-51.

$23 \mathrm{Hu}$ J, Kind AJH, Nerenz D. Area deprivation index predicts readmission risk at an urban teaching hospital. Am J Med Qual 2018;33:493-501.

24 Kind AJH, Jencks S, Brock J, et al. Neighborhood socioeconomic disadvantage and 30-day rehospitalization: a retrospective cohort study. Ann Intern Med 2014;161:765-74.

25 Jencks SF, Schuster A, Dougherty GB, et al. Safety-Net hospitals, neighborhood disadvantage, and readmissions under Maryland's all-payer program: an observational study. Ann Intern Med 2019;171:91-8 